\title{
Heredofamilial deficiency of monocyte esterase in patients with rheumatoid arthritis
}

\author{
A L Bell, G M Markey, M D McCaigue, D Middleton, J A McCormick, A G Wilson, \\ T C M Morris
}

\begin{abstract}
Deficiency of the monocyte ectoenzyme non-specific esterase is described in a heredofamilial pattern in four patients with rheumatoid arthritis. No association with HLA status or rheumatoid factor seropositivity was found.
\end{abstract}

Cytochemical staining for the ectoenzyme ${ }^{1}$ monocyte serine esterase or non-specific esterase has become a generally recognised laboratory technique for the identification of cells of the mononuclear phagocyte system. Five carboxyl esterase isoenzymes capable of reacting with standard substrates have been described in monocytes. ${ }^{2}$ Monocyte esterase deficiency in blood monocytes or their circulating precursors from patients with rheumatoid arthritis (RA) has been described, ${ }^{3}$ though no suggestion of familial deficiency has been made. We have previously reported an association between familial monocyte esterase deficiency and malignant disease. ${ }^{45} \mathrm{We}$ therefore carried out family studies in a small number of patients with coexistent RA and monocyte esterase deficiency to examine the possibility that the trait is familial, and to examine relations with arthritis, HLA status, and rheumatoid factor.

\section{Patients and methods}

In a large outpatient survey seven patients with rheumatoid arthritis were found to have monocyte esterase deficiency, ${ }^{5}$ three of whom were selected for study. An additional monocyte esterase deficient patient identified during hospital admission was also selected. All patients fulfilled the diagnostic criteria for $\mathrm{RA}^{6}$ and three were seropositive for rheumatoid factor. One seropositive patient also had psoriasis and one had severe nodular systemic RA with active necrotising cutaneous vasculitis. No other patient had nodules. Repeat blood samples were stained for up to six months after identification and the deficiency was shown to be constant.

Family members were initially contacted by index patients. After informed consent, each patient and their relative were examined for evidence of arthritis and provided a blood sample for monocyte cytochemistry, tissue typing, and rheumatoid factor assay.

\section{CYTOCHEMISTRY}

Cytocentrifuge preparations of Ficoll-Hypaque density gradient separated mononuclear cells were stained for monocyte esterase with $\alpha$ - naphthyl acetate as substrate ${ }^{7}$ at $\mathrm{pH} 5 \cdot 8$ using a batch technique. Monocyte esterase positive control monocytes were included in all batches stained. Lymphocyte dot positivity provided an on slide control of the sensitivity of the procedure. Results were independently verified by a 'blinded' observer in a separate laboratory (department of haematology, Belfast City Hospital). Pronounced monocyte esterase deficiency was considered to be present if greater than $85 \%$ of monocytes failed to stain $^{5}$; partial deficiency was assumed if $50-85 \%$ of cells were esterase negative. Wright's stained cytocentrifuge slides were used to confirm that the cells examined were mature monocytes. Two of the index patients, tested repeatedly over an 18 month period, were classified as having pronounced monocyte esterase deficiency on each occasion.

\section{TISSUE TYPING}

Samples for full HLA haplotypes were obtained from three index patients and their relatives. Tissue typing was performed using a standard microcytotoxicity test. For HLA-DR typing B lymphocytes were isolated using magnetic beads coated with a monoclonal antibody to HLA class II.

\section{RHEUMATOID FACTOR}

Standard RA latex (Wellcome Laboratories, Beckenham, Kent, United Kingdom) and RA particle agglutination test kits (Serodia-RA, Fujirebio, Japan) were used for the determination of rheumatoid factor.

\section{Results}

Thirty seven subjects were studied ( 33 family members and four propositi). Twelve (32\%) showed pronounced monocyte esterase deficiency and six (16\%) partial deficiency. The family pedigrees (fig) showed enzyme deficiency in siblings and relatives of both sexes. Of the 12 offspring of the four propositi (two men, two women), 10 were investigated for monocyte esterase deficiency. Of these 10 , three had complete and three partial monocyte esterase deficiency. Of the 21 sibs of the propositi, 18 were investigated of whom seven had total or partial monocyte esterase deficiency. Of the eight parents of the propositi, only three were available for testing; two refused testing and the only one tested had monocyte esterase deficiency. These findings are consistent with autosomal dominant inheritance. 

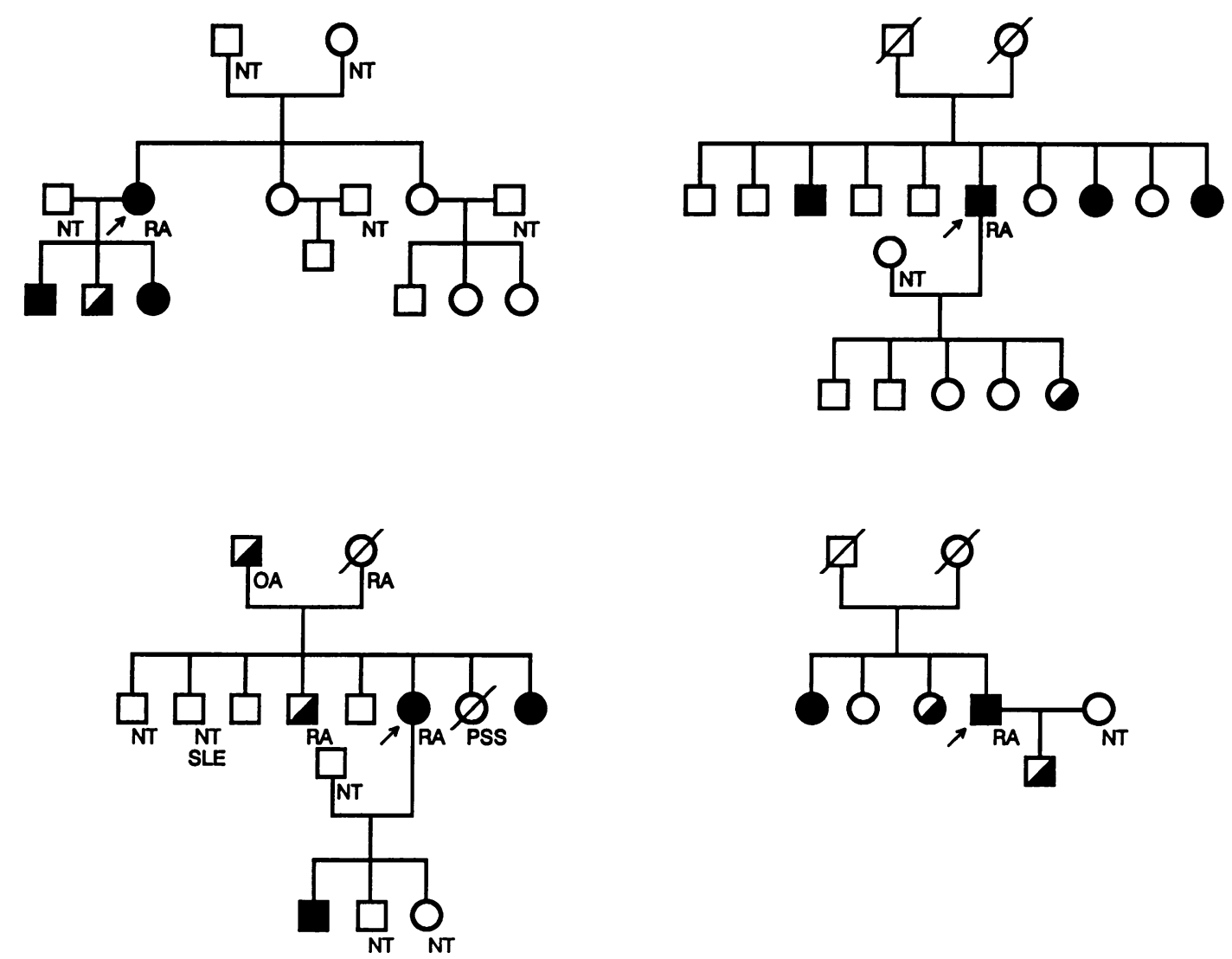

Family pedigrees showing complete and partial monocyte esterase deficiency. $\quad \nabla=$ propositus; $N T=$ not tested; $\square=$ male; $\mathrm{O}=$ female; $\mathbf{O}=$ partial monocyte esterase deficiency; $\mathbf{O}=$ complete monocyte esterase deficiency; $\not \varnothing=$ deceased; $R A=$ rheumatoid arthritis; $P S S=$ progressive systemic sclerosis; and $S L E=$ systemic lupus erythematosus.

One family showed a strong history of autoimmune rheumatic disease (fig), and rheumatoid factor positivity was found in two affected relatives (one with RA) and three index patients. Tissue typing showed that all index patients were HLA-DR4 positive and one was homozygous. There was no suggestion of coinheritance of HLA haplotypes with monocyte esterase deficiency, and no association of enzyme deficiency with the presence of rheumatoid factor.

\section{Discussion}

Cytocentrifuge preparations of mononuclear cells obtained over Ficoll-Hypaque from patients without haematological diseases contain only lymphocytes and monocytes. The characteristic morphology of mature monocytic populations seen on monocyte esterase stained slides (whether monocyte esterase positive or negative) and Wright's stained slides is sufficient for the identification of most of this population. It has previously been shown that the cells which have a typical morphology on Wright's stained preparations but are monocyte esterase negative are monocytes and have positive reactions with antibodies to muramidase and $\alpha-1$-trypsin. ${ }^{8}$ The identification of monocyte esterase deficiency in this study is therefore valid. The study showed heredofamilial monocyte esterase deficiency occurring in patients with RA. In agreement with other hereditary esterase deficiencies an autosomal pattern of inheritance was found.

Hurst et $a l^{3}$ described a decrease in the proportion of esterase positive monocytes correlating with a decrease in $\mathrm{C} 3 \mathrm{~b}$ mediated phagocytosis in 13 patients with RA who had active vasculitis or extra-articular disease manifestations. Patients with inactive vasculitis or multiple nodules did not show a discrepancy. The appearance of large monocyte esterase negative cells during active disease appears to correlate with the findings of Horwitz and Steagall, who studied large atypical mononuclear cells occurring in the peripheral blood of patients with rheumatic diseases. ${ }^{9}$ The latter study showed that one of nine of these immature cells became mature monocytes. Our findings are clearly different from and should not be confused with the findings of these studies. Only mature monocytes were considered in our study; the criteria for diagnosis of severe monocyte esterase deficiency was that greater than $85 \%$ of mature monocytes were esterase negative, the deficiency was constant (not fluctuating over months or years), and it was clearly a familial trait.

In our previously reported survey, ${ }^{5}$ the occurrence of severe monocyte esterase deficiency was $0.8 \%$ (four of 474 subjects) in the normal population. In this survey 3172 patients with non-malignant diseases and 808 patients with malignant diseases were investigated. Of the group with non-malignant diseases only patients with renal disease $(n=291)$ showed a significant increase $(3.8 \% ; p=0.0112)$ in the incidence of the anomaly; $50 \%$ of the monocyte esterase deficient patients had renal disease of autoimmune aetiology. Only two groups of patients with autoimmune disease were well 
defined, namely those with RA $(n=218)$ and those with autoimmune haematological diseases $(n=22)$. In each of these groups there was a trend towards an increased incidence of the anomaly $(3.2 \%(p=0.0547)$ and $9 \%(p=0.0506)$ respectively). There was also a significant increase $(6.2 \% ; p<0.001)$ in monocyte esterase deficiency in patients with lymphoproliferative malignancies. In view of the well described association between autoimmune diseases, including $\mathrm{RA}$, and lymphoproliferative malignancy, ${ }^{10}$ the findings of an increased association or trend towards an increased association of this trait (which has been familial whichever group of patients were investigated) with these diseases in our survey was striking, and may suggest a link between heredofamilial monocyte esterase deficiency and these diseases.

The familial trait could represent an additional genetic influence on the development or expression of RA. In this study it did not closely relate to the possession of HLA-DR4. In our outpatient survey ${ }^{5}$ of seven monocyte esterase deficient patients with rheumatoid arthritis, two had systemic vasculitis (29\%) compared with 11 of 211 monocyte esterase positive RA patients $(5 \%)$. It is therefore possible that the enzyme deficiency may predispose to severe systemic involvement in RA.

The biological role of monocyte esterase is uncertain. In studies with a specific monocyte esterase inhibitor Oertel et al suggested that the enzyme is required for spontaneous monocyte cytotoxicity to K562 cell lines. ${ }^{2}$ No effects on monocyte adhesion, spreading, or latex particle phagocytosis were found. Moreover, esterase negative monocytes have been shown to be unable to lyse K562 cells when stimulated by lactoferrin. ${ }^{11}$ A possible link between monocyte esterase deficiency and defective monocyte $\mathrm{C} 3 \mathrm{~b}$ mediated phagocyte function ${ }^{3}$ has been outlined above.

It is possible that familial monocyte esterase deficiency predisposes patients with RA to lymphoproliferative malignancy. Markey et al ${ }^{4}$ have shown an increased occurrence of the deficiency in patients with non-Hodgkin's lymphoma and B cell chronic lymphocytic leukaemia, haematological malignancies found with increased occurrence in RA. ${ }^{10}$ It has been suggested that diminished natural killer cell cytotoxicity in RA predisposes to the development of B cell chronic lymphocytic leukaemia ${ }^{12}$ and non-Hodgkin's lymphoma ${ }^{13}$; these papers did not consider monocyte cytotoxicity.

Finally, the possibility exists that monocyte esterase deficiency is merely a coincidental finding in these patients with RA. Further studies to define the functional consequences for monocytes of heredofamilial esterase deficiency in patients with RA have begun.

We are grateful to Professor N Nevin, Professor of Medical Genetics, The Queen's University of Belfast for helpful comments on the family pedigrees; Mr T Ferguson and other staff of the laboratories, Musgrave Park Hospital for valuable technical assistance; and Mrs Moya Maguire for efficient preparation of the manuscript.

1 Bozdech M J, Bainton D F. Identification of $\alpha$-naphthyl butyrate esterase as a plasma membrane ectoenzyme of monocytes and as a discrete intracellular membranebounded organelle in lymphocytes. $\mathcal{F}$ Exp Med 1981; 153: 182-95.

2 Oertel J, Majner G, Kastner M, Huhn D. The relevance of $\alpha$ naphthyl acetate esterases to various monocyte functions. naphthyl acetate esterases to various
$\mathrm{Br} \mathcal{F}$ Haematol $1985 ; 61: 717-26$.

3 Hurst N P, Nuki G, Wallington T. Functional defects of monocyte $\mathrm{C} 3 \mathrm{~b}$ receptor-mediated phagocytosis in rheumatoid arthritis (RA): evidence for an association with the appearance of a circulating population of nonspecific esterase-negative mononuclear phagocytes. Ann Rheum Dis 1983; 42: 487-93.

4 Markey G M, Morris T C M, Alexander M D. Monocyte esterase? A factor involved in the pathogenesis of lymphoproliferative neoplasia. Leukaemia 1987; 1: 236-9.

5 Markey G M, McCormick J A, Morris T C M, et al. Monocyte esterase deficiency in malignant neoplasia. $\mathcal{f}$ Clin Pathol 1990; 45: 282-6.

6 Arnett F C, Edworthy S M, Bloch D A, et al. The American Rheumatism Association 1987 revised criteria for the classification of rheumatoid arthritis. Arthritis Rheum 1988; 31: $315-24$

7 Yam L T, Li C Y, Crosby W H. Cytochemical identification of monocytes and granulocytes. Am $\mathcal{F}$ Clin Pathol 1971; 55: 283-90

8 Markey G M, Alexander H D, McConnell R, Kyle A, Morris T C M, Robertson J H. Hereditary monocyte esterase deficiency. Br $\mathcal{F}$ Haematol 1986; 63: 359-63.

9 Horwitz D A, Steagall R V Jr. The development of macrophages from large mononuclear cells in the blood of patients with inflammatory disease. $\mathcal{F}$ Clin Invest 1972; 51: 760-8.

10 Symmons D P M, Ahern M, Bacon P A, et al. Lymphoproliferative malignancy in rheumatoid arthritis: a study of 20 cases. Ann Rheum Dis 1984; 43: 132-5.

11 McCormick J A, Markey G M, Morris T C M, Auld P W, Alexander $\mathrm{H} \mathrm{D}$, et al. Lactoferrin inducible monocyte cytotoxicity defective in esterase deficient monocytes. $B r \mathcal{F}$ Haematol 1991; 77: 287-90.

12 Taylor H G, Nixon N, Sheeran T P, Dawes P T. Rheumatoid arthritis and chronic lymphatic leukaemia. Clin Exp Rheumatol 1989; 7: 529-32.

13 Potter D, Madhok R, Capell H. Non-Hodgkin's lymphoma in rheumatoid arthritis [leader]. Ann Rheum Dis 1991; 50: 275-6. 